
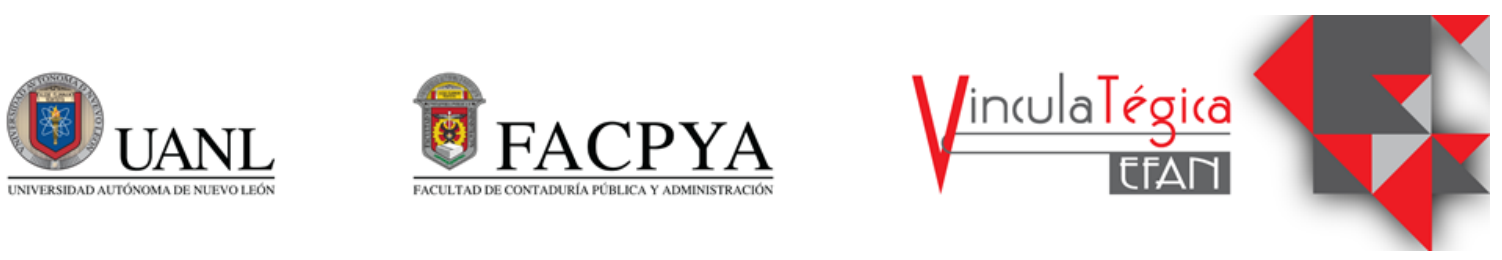

\title{
Ubicuidad y diversificación como factores de complejidad económica en Sinaloa
}

\author{
Víctor García Valenzuela ${ }^{1}$, Missael Ruiz Corrales ${ }^{2}$ y Angélica Reyes Mendoza ${ }^{3}$ \\ ${ }^{1}$ Instituto Tecnológico de Sonora, victor.garcia@potros.itson.edu.mx, Calle 5 de Febrero 818 Centro. CP \\ 85000 Cd. Obregón Sonora, 6444109000 \\ ${ }^{2}$ Universidad Autónoma de Baja California, ruizcmissael@gmail.com, Delegación Mesa de Otay CP22390 \\ Tijuana, Baja California, 6649797500 \\ ${ }^{3}$ Universidad Autónoma de Baja California, angelita@uabc.edu.mx, Delegación Mesa de Otay CP22390 \\ Tijuana, Baja California, 6649797500
}

Información del artículo revisado por pares

Fecha de aceptación: junio-2021

Fecha de publicación en línea: diciembre-2021

DOI: https://doi.org/10.29105/vtga7.1-94

\section{Resumen}

El objetivo del artículo es determinar el nivel de complejidad mediante el cálculo de diversidad y ubicuidad de los 5 municipios más importantes de Sinaloa, México. Se empleó la información oficial del Atlas de Complejidad Económica de México para aplicar el método de matrices binarias con la fórmula de ventajas comparativas y el cálculo de las variables diversidad y ubicuidad según la metodología teórica. Los resultados indican que Culiacán con diversidad de 131 y con 1,5124 en ubicuidad, es el municipio con mayor complejidad económica y el más competitivo en cuanto a su desempeño de aprovechamiento de recursos, por lo que se genera nuevo y productivo conocimiento acerca de la complejidad productiva de cada uno de los principales municipios de Sinaloa. La metodología aplicada mediante los cálculos realizados muestra adecuadamente el nivel de complejidad económica de los municipios más importantes de Sinaloa, México.

\begin{abstract}
The objective of the article is to determine the level of complexity by calculating the diversity and ubiquity of the 5 most important municipalities in Sinaloa, Mexico. The official information of the Atlas of Economic Complexity of Mexico was used to apply the method of binary matrices with the formula of comparative advantages and the calculation of the variables diversity and ubiquity according to the theoretical methodology. The results indicate that Culiacán, with diversity of 131 and with 1,5124 in ubiquity, is the municipality with the greatest economic complexity and the most competitive in terms of its performance in the use of resources, thus generating new and productive knowledge about productive complexity. from each of the main municipalities of Sinaloa. The methodology applied through the calculations carried out adequately shows the level of economic complexity of the most important municipalities of Sinaloa, Mexico.
\end{abstract}

Palabras clave: Complejidad, Diversidad, 
Ubicuidad.

\section{INTRODUCCIÓN}

Tanto las economías como las empresas se adentran a una etapa de tecnología, e información, que, con la nueva era del conocimiento, el desarrollo empresarial está en función a la generación y aumento del conocimiento productivo en las entidades económicas. Estos conocimientos son reflejados en la complejidad económica a través de las variables diversidad y ubicuidad, las cuales describen la estructura productiva de una economía, lo cual se manifiesta en el nivel de riqueza y crecimiento de las mismas. Las economías poco desarrolladas tienden a producir bienes y servicios de poca competitividad con tecnología y conocimiento sencillo y básico, por otro lado, las economías altamente desarrolladas tienden a generar bienes y servicios con mayor valor agregado mediante la aplicación de tecnología y conocimiento más complejo según Pérez, Salazar y Mendoza (2019).

Hausmann e Hidalgo (2009) son los creadores de la teoría de complejidad económica mediante la cual proponen una metodología para determinar el crecimiento de una economía bajo una perspectiva macroeconómica, aunque el Producto Interno Bruto (PIB) normalmente es utilizado para determinar dicho crecimiento, este solo se basa en la productividad y la teoría de complejidad económica va más allá de la simple productividad, posee un modelo de medición productiva que está basado en la complejidad y sofisticación de los productos conforme a dos variables: diversidad $y$ ubicuidad. Los modelos complejos en economía poseen un mayor grado de explicación del comportamiento real de una entidad económica, ya que al ser modelos novedosos poseen una perspectiva de la nueva economía, al poseer vectores más flexibles para la interpretación del comportamiento económico, los hace una herramienta más eficiente que los modelos tradicionales.

A través de una publicación del Banco de México (BANXICO) los autores Gómez,
Keywords: Complexity, Diversity, Ubiquity. JEL: O11, O41, R11

Chavez y Mosqueda (2016) mediante la metodología propuesta por Hausmann e Hidalgo (2009) realizan los cálculos de complejidad económica a nivel nacional y así logran estadísticamente comprobar la diferencia de los estados de la República Mexicana en cuanto a su especialización de actividades económicas y evidentemente en la complejidad económica de los mismos estados, de esta forma se determina como la desigualdad de cada entidad federativa está en función de las actividades en las cuales se especializan y la diversificación de sus productos que ofrecen al mercado. En sus resultados los autores Gómez, Chavez y Mosqueda (2016) muestran la evolución de mayor a menor de la complejidad económica de cada uno de los estados en comparación con todas las entidades federativas que conforman el país, los estados que encabezan la lista de complejidad económica son Nuevo León, Coahuila, Distrito Federal y Chihuahua y los estados con menor nivel de complejidad económica son Oaxaca, Nayarit, Guerrero y Chiapas. Los estados que encabezan la lista de complejidad económica son los que tienden evolucionar o crecer su capacidad para elaborar productos cada vez más complejos, los estados que están al final de la lista son aquellos que producen productos con un nivel de ubicuidad muy alto por lo tanto no son competitivos, con base a este supuesto, se puede percatar que el amplio volumen de productividad no garantiza el crecimiento económico.

El propósito de esta investigación es calcular las variables diversidad y ubicuidad de la complejidad económica de los cinco municipios más representativos del estado de Sinaloa los cuales son Ahome, Culiacán, Mazatlán, Navolato y Mochis con el fin de conocer el nivel de complejidad y competitividad bajo una perspectiva agregada $\mathrm{y}$ diferente para medir el desempeño y crecimiento económico.

A lo largo de las últimas décadas, las economías se han desarrollado y crecido con 
relación a su capacidad para hacerlo, y es bajo esta misma capacidad de crecer el por qué la riqueza y prosperidad no se pueden apreciar de forma equitativa para todas las economías. La desigualdad de distribución de recursos ha ocasionado inestabilidad económica y política en los países principalmente de américa latina.

Según las publicaciones del PIB por el Instituto Nacional de Estadística, Geografía e Informática [INEGI] (2015) el 60\% del indicador macroeconómico es generado por sólo 8 estados los cuales son Estado de México, Guanajuato, Nuevo León, Jalisco, Coahuila, Veracruz, Puebla y Tamaulipas, el otro $40 \%$ es generado por los otros 24 estados de manera conjunta, la participación de los estados en el PIB nacional es un claro ejemplo de desigualdad de las economías en cuanto su capacidad para generar, crecer y desarrollar recursos o beneficios. De forma más específica, las actividades económicas también son generadas de forma divergente por los estados, el $69.6 \%$ de la actividad primaria es generada por 7 estados (Sinaloa, Sonora, Michoacán, Veracruz, Chihuahua, Jalisco y Guanajuato), el $71.6 \%$ de la actividad secundaria es generado por 7 estados (Estado de México, Sonora, Tabasco, Ciudad de México, Campeche, Nuevo León y Jalisco) y el $64.3 \%$ de la actividad terciaria es generado por solo 7 estados del país ( Coahuila, Estado de México, Nuevo León, Tamaulipas, Puebla, Jalisco y Ciudad de México). Es comprensible que no todos los estados están especializados en todas las actividades económicas, pero de 32 estados en cada actividad solo 7 son capaces de aportar más del $60 \%$ de dichas actividades, bajo este argumento, las principales entidades de investigación y ciencia intentan reconocer la causa de esta desigualdad, si bien no todas las economías poseen las mismas capacidades para crecer y desarrollarse, determinar el cómo poder igualar dicha capacidad se ha vuelto en una incógnita importante en los últimos años.

El Instituto Mexicano para la Competitividad [IMCO] (2016) en una de sus publicaciones determinan que, en México, aunque se hace referencia de un único país consolidado, en realidad existe bastante diversidad en el mismo y no solo cultural o geográfica, sino económica. Ya que IMCO menciona que en México se viven dos versiones diferentes, está el México del norte donde los estados tienen un crecimiento de forma sostenida donde atraen inversión y generación de empleos formales, y también existe el México del sur conformado por los estados en rezago económico donde los niveles de pobreza están arriba del promedio nacional.

Aquí surge la relevancia de la presente investigación, al analizar los municipios bajo una perspectiva diferente bajo los supuestos metodológicos de una teoría macroeconómica se obtiene una nueva interpretación del comportamiento económico el cual puede determinar la razón de la diversidad económica entre las economías analizadas que según la teoría de complejidad económica (Donoso y Martín, 2017) esto dependerá de los resultados de los cálculos de las variables diversidad y ubicuidad

Analizar a los municipios bajo esta metodología permite conocer el nivel de competitividad de los mismos partiendo del cálculo de las dos variables de la teoría de complejidad económica. El índice de diversidad determinará la cantidad de sectores en los cuales los municipios se encuentran especializados en comparación con el resto en función a las Ventajas Comparativas Reveladas (VCR) encontradas en las actividades económicas de cada municipio. El índice de ubicuidad determinará lo complejo o imitable que es el proceso productivo de cada uno de los sectores en los cuales se especializa, determinando de esta manera cuantos municipios son capaces de competir dentro del mismo sector en función a los municipios que presentan VCR en el mismo sector en el cual están especializados.

De acuerdo con la teoría, los municipios que obtengan indicadores de diversidad y ubicuidad más altos obtendrán un mayor nivel de complejidad económica. Entre mayor sea el nivel de complejidad de la entidad económica, mayor será la competitividad del municipio, y partiendo de los supuestos de la teoría, mayor será su red de conocimiento y su ecosistema de innovación, es así como se determina el nuevo indicador de 
desempeño para las economías analizadas.

Posteriormente, se clasificaron los municipios con base a sus niveles de complejidad económica, con la finalidad de obtener un clasificador basado en la teoría de complejidad económica que logra interpretar el comportamiento económico de los municipios bajo una perspectiva diferente a las utilizadas generalmente. Asimismo, el cálculo de los indicadores y la clasificación de los municipios permiten crear supuestos bajo los cuales pueden estructurarse las mediciones de complejidad de los municipios para generar restricciones y supuestos en la bondad de utilizar la teoría de complejidad económica como herramienta para las economías para clasificar y medir el desempeño de estas.

Esta investigación busca medir a los municipios mediante las variables de la teoría de complejidad económica (diversidad y ubicuidad) aplicando el método de matrices binarias a través de la fórmula de $\mathrm{VCR}$ al igual que Gómez, Chavez y Mosqueda (2016) por parte de BANXICO. Por lo que se plantea el siguiente objetivo de investigación:

- Calcular el nivel de complejidad económica de los municipios a través de las variables diversidad y ubicuidad.

\section{MARCO TEÓRICO}

La teoría de recursos y capacidades de Penrosee (1959) citado por Sánchez y Herrera (2016) aborda la respuesta a el porque las empresas al igual que las economías son diferentes entre sí, aunque estas puedan pertenecer al mismo giro o sector, y esto es debido a que todas las entidades económicas poseen recursos y capacidades diferentes. Se entiende como recursos aquellos internos como externos que la empresa posee para poder desarrollar capacidades dinámicas diferentes que le otorguen valor agregado a un bien o servicio, el óptimo aprovechamiento de los recursos permite diferenciar a las empresas.

Esta diferencia de posesión de recursos es debido a las dos características que tienen los recursos: heterogeneidad e imperfecta movilidad. La heterogeneidad hace referencia a que no todos los recursos son iguales ya que, aunque una economía pueda llegar a poseer el mismo recurso, esto no quiere decir que esté desarrollado y aprovechado de la misma forma y es debido a la segunda característica, la movilidad imperfecta, la cual indica que no todos los recursos están disponibles para todas las entidades económicas, un ejemplo muy claro de esto es el petróleo (Vargas y Cano, 2016). La clasificación de los recursos es una herramienta clave para lograr identificarlos eficientemente, estos se dividen en dos categorías: tangibles e intangibles. Son los intangibles los esenciales en la teoría de complejidad económica, ya que de acuerdo con Nonaka y Takeuchi (1995) el único recurso capaz de generar ventajas competitivas sostenidas y perdurables en el tiempo es el más importante para las empresas: el talento humano.

Tanto los recursos como las capacidades son esenciales para poder generar ventajas competitivas en una entidad económica, estas según Porter (1991) citado por Vega y Schmutzler (2017) son el valor agregado que es generado por la empresa en un bien o servicio para el cliente, justificando el hecho de que el cliente pague un precio más elevado por dicho bien o servicio con beneficios particulares al resto del mercado. El implementar de manera óptima las ventajas competitivas permite aprovechar de forma eficiente las capacidades y recursos de una economía, esto tiende a incrementar la mejora en la estructura organizacional tanto en los procesos productivos, financieros $\mathrm{o}$ administrativos. Si una economía quiere que su ventaja sea perdurable y persista en el mercado, esta debe ser única e inimitable conforme a Garcia, Meza y Pedraza (2018).

La teoría de complejidad económica (Gómez y Molina, 2018) es utilizada para determinar el desempeño de una economía mediante una perspectiva macroeconómica enfocada en cuantos tipos de productos ofrece al mercado y la complejidad de los procesos productivos de los mismos. El concepto clave dentro de esta teoría es el conocimiento productivo, ya que este se refiere a con base a qué conocimientos están realizados los productos, apartando las materias primas o 
insumos de un producto y enfocándose en las ideas con las cuales se realiza el producto. Mientras menor sea la complejidad de los procesos productivos de un bien o servicio, menor conocimiento será implementado para elaborar ese producto, mientras mayor complejidad tenga un proceso productivo, mayor será el conocimiento implementado y el valor agregado generado a ese producto, por lo tanto, mayor probabilidad de que es economía expanda su ecosistema de innovación y su red de conocimiento.

$\mathrm{Si}$ bien el PIB es el indicador generalmente utilizado para determinar el desempeño y crecimiento de un país (INEGI, 2013), la teoría de complejidad económica no degrada la funcionalidad de este indicador como herramienta macroeconómica, si no que va más allá de la productividad de una entidad económica, los autores de esta teoría argumentan que para determinar de forma real el desempeño y desarrollo de una economía es analizando la capacidad de diversificar la estructura productiva y el aumento de conocimiento productivo de una entidad económica y esto es posible mediante el cálculo de dos variables: diversidad y ubicuidad.

Si bien el conocimiento productivo es el concepto clave para la teoría de complejidad económica, la transferencia del mismo es aún más importante ya que es mediante este proceso como este conocimiento se moviliza dentro de las economías y a su vez va evolucionando. Pero no es cualquier tipo de conocimiento el que se refiere esta teoría, dejando de lado el conocimiento común y haciendo énfasis en el conocimiento "táctico", este tipo de conocimiento es el que permite a los individuos en pasar de ideas a hechos, o en el caso de las economías el que les permite pasar de ideas a productos nuevos, innovando así en el mercado mediante la aplicación de mayor conocimiento en los procesos productivos y convirtiéndolos cada vez más complejos según Lara, Tosi y Altimiras (2018).

En la teoría de complejidad económica, si la percepción de una economía en cuanto la generación de conocimiento productivo es mayor, mayor complejidad económica poseerá, esto hace referencia a que las economías no solo son evaluadas por los productos que ofrecen al mercado, sino también por la posible ocurrencia de las economías en presentar oportunidades de crecer su red de conocimiento y expandir su ecosistema de innovación, dictaminando así que los productos funcionan como vehículos para el conocimiento, esto es el cimiento de la complejidad económica conforme a García, Ruiz, López y Placencia (2019).

Tanto la diversidad como la ubicuidad son variables determinantes de la complejidad económica, ambas son sumamente necesarias para llevar a cabo los cálculos necesarios en busca de los resultados de complejidad, no obstante, la misma búsqueda de diversificación de los productos, surge el decremento de ubicuidad de los mismos dentro del mismo proceso de especialización de las actividades económicas, por lo tanto, una economía diversificada tiende a producir bienes y servicios poco ubicuos o poco imitables, Pérez, Moheno y Salazar (2019) denominan la diversidad cuando una entidad económica se introduce a líneas nuevas de producción fomentado el desarrollo de la empresa. Según Grant (1991) citado por Tortoriello (2015) cuando los procesos productivos tienden a ser más complejos, estos conllevan mayor conocimiento aplicado, por lo tanto, de los mismos recursos puede iniciar el proceso de crear nuevos productos o bien, especializarse en los mismos haciendo que sus productos tengan mayor valor agregado para el cliente disminuyendo el grado de ubicuidad de los mismos.

Diversidad es la capacidad de las economías para aprovechar sus recursos y crear productos nuevos, conforme sea mayor el número de productos que ofrece al mercado una economía, mayor será su nivel de complejidad económica. La ubicuidad es la capacidad de las economías en convertir sus productos poco imitables mediante la sofisticación de los procesos productivos, mientras menos economías produzcan dicho bien o servicio, mayor será la complejidad económica (Hausmann, Cheston y Santos, 2015).

Es desde la competencia de mercado 
donde se propicia la necesidad de las economías en diversificar y disminuir la ubicuidad de sus bienes y/o servicios mediante la aplicación de ventajas competitivas sostenidas a través de estrategias empresariales para así ganar la preferencia de los clientes de un mercado en específico. Las empresas buscan la diversificación y la poca ubicuidad para incentivar su impacto en el mercado, en este proceso existe algo llamado innovación lo cual, aunque es un desafío para las empresas, orientan al crecimiento de la empresa.

Analizar a las economías mediante la diversidad y la ubicuidad, permite interpretar el comportamiento de la productividad de una economía no solo conforme a su nivel de producción, también con base a lo sofisticado que es realizar un bien o servicio. Aquella economía que produce productos complejos tiende a situarse en un entorno desarrollado y de innovación a diferencia a las economías poca complejas, por lo tanto, el mismo desarrollo económico es resultado del proceso de generación y acumulación de conocimiento productivo, amplificando la estructura productiva de las entidades económicas desarrollando mayores capacidades productivas con base a bienes y servicios más diversificados y menos ubicuidad

\section{MÉTODO}

La investigación en función al objetivo se considera que posee un análisis cuantitativo con diseño no experimental ya que no se manipuló ninguna variable planteada. $\mathrm{La}$ información se obtuvo de una fuente secundaria de origen de corte transversal debido a que se recolectó en un tiempo único.

Los 5 sujetos analizados son los 5 municipios más representativos del estado de Sinaloa, México, los cuales son Culiacán, Ahome, Guasave, Mazatlán y Navolato, en función al número de personal ocupado según INEGI (2019) por efecto de la metodología de las ventajas comparativas reveladas (VCR).

La fuente de información llamada "Atlas de Complejidad Económica" son el resultado de un trabajo que realizó el Centro de Desarrollo Internacional de la Universidad de Harvard para calcular el ICE de México en conjunto con la Secretaría de Hacienda y Crédito Público (SHCP) y el Centro de Investigación y Docencia Económica (CIDE) en el 2014, se utiliza esta fuente debido a que es la única que brinda la información necesaria para calcular las variables de la complejidad económica. En esta base de datos encontramos la proporción de personal ocupado de cada uno de los municipios en cada uno de los sectores en los cuales se desempeñan, por ende, el total de sectores o actividades económicas que se llevan a cabo en el estado de Sinaloa las cuales son 309.

Para obtener la medida de complejidad económica de una entidad económica se puede utilizar dos tipos de datos: exportaciones o el empleo, que en este caso fue por el número de personal ocupado. La metodología se distribuye en los siguientes tres pasos, mismos utilizaron Gómez, Chávez y Mosqueda (2016) propuesta por Hausmann e Hidalgo (2009) quienes son los mismos autores de la teoría.

1. Se calculó calcula las VCR de cada uno de los municipios con relación a cada uno de los sectores donde tienen participación económica en función a la proporción de personal ocupado tanto del municipio, del sector y del estado.

2. Se calculó la variable diversidad mediante una matriz binaria que expresa las actividades económicas en las cuales se especializa cada municipio.

3. Se calculó la variable ubicuidad mediante una matriz binaria según la localización de las diversas actividades económicas de cada municipio.

La matriz binaria antes mencionada expresa en qué sectores se especializa cada municipio, para esto se utiliza el método de ventajas comparativas reveladas (VCR) el cual sigue la siguiente fórmula: 
Donde:

$P s, a=$ el número de personas empleadas en la actividad económica $\alpha$ en el municipio $\mathrm{S}$.

$\sum_{\alpha=1}^{n} \quad p s, a=$ el número total de personal ocupado en el municipio $\mathrm{S}$.

$\sum_{s=1}^{x} \quad p s, a=$ el número de personal ocupado en el sector $\alpha$ en el estado.

$\sum_{s=1, \alpha=1}^{s=x, \alpha=n} \quad p s, a=$ el número total de personas empleadas en el estado.

Se propone como umbral que VCR

$\geq 1$, esto refiere a que el municipio analizado "s" estará especializado en el determinado sector "a" si la misma proporción de personal ocupado en este sector con relación al total de personal ocupado en el municipio es igual o mayor a la proporción análoga del estado.

Consecuente a esto se aplican las fórmulas de diversidad y ubicuidad utilizadas por Gómez, Chávez y Mosqueda (2016) mismas que proponen los autores de la teoría Hausmann e Hidalgo (2009).

$$
\begin{aligned}
& K s, o=\sum_{\alpha=1}^{n} \quad M s, a=\text { Diversidad } \\
& K \alpha, o=\sum_{s=1}^{x} \quad M s, a=\quad \text { Ubicuidad }
\end{aligned}
$$

Diversidad es la sumatoria de todos aquellos sectores en los cuales se especializa cada uno de los municipios analizados con base a su VCR. Diversidad es un vector de $1 * \mathrm{n}$ donde las mismas entradas pueden ser de 1 a n, que en este caso sería hasta 309 al ser el número de sectores que se desempeñan en todo el estado de Sinaloa, esto indicará la cantidad de las actividades económicas que se especializa el municipio analizado.

Diversidad es la sumatoria de todos aquellos municipios que realizan la misma actividad económica o sector analizado con base al VCR de los municipios. Ubicuidad es un vector de $1^{*} \mathrm{X}$, cuyas entradas pueden ser de 1 a $X$, que en este caso sería hasta 5 al ser 5 los municipios analizados, esto indicará la cantidad de municipios que desempeñan la misma actividad económica en la cual se especializan.

La medida de complejidad económica está sujeta al mayor nivel de diversidad que obtiene el municipio según el número de sectores en los cuales se especializa y al menor nivel de ubicuidad que obtiene el municipio el número de municipios que están especializados en el mismo sector.

\section{RESULTADOS}

Posteriormente de los cálculos correspondientes para cada una de las variables (diversidad y ubicuidad) que determinan la complejidad económica se presentan los resultados de la aplicación de la metodología propuesta por Hausmann e Hidalgo (2009) en función al número de personal ocupado por municipio, lo mismo que realizaron Gómez, Chávez y Mosqueda (2016) mediante Banxico.

Conforme a los cálculos realizados, en primera instancia se determinó la variable diversidad el cual indica el número de sectores en los cuales se especializa y desarrolla cada municipio, esta cantidad proviene de la sumatoria de aquellos sectores en los cuales los municipios presentan una VCR en comparación con el resto de los municipios analizados. La tabla 1 indica los resultados de la variable diversidad para cada uno de los municipios.

Tabla 3: Diversidad de los municipios estudiados.

\begin{tabular}{ccc}
\hline Municipio & Diversidad & Representación \\
\hline Ahome & 116 & $37,54 \%$ \\
Culiacán & 131 & $42,39 \%$ \\
Mazatlán & 118 & $38,19 \%$ \\
Guasave & 70 & $22,65 \%$
\end{tabular}




\begin{tabular}{ccc} 
Navolato & 39 & $12,62 \%$ \\
\hline & Fuente: Elaboración propia.
\end{tabular}

La tabla 3 muestra los resultados de la variable diversidad correspondiente a los 5 municipios analizados. El municipio que obtuvo mayor diversidad es Culiacán con una cantidad de 131 lo cual es una representación de un $42,39 \%$ de los 309 sectores totales que se desarrollan en el estado de Sinaloa. En segundo lugar, se encuentra Mazatlán con tan solo 2 sectores más que Ahome con una cantidad de 118 sectores y 116 para Ahome lo cual es una representación del $38,19 \%$ y $37,54 \%$ del total de sectores. Posteriormente está el municipio de Guasave con una cantidad de 70 sectores los cuales representan un $22,65 \%$ y por último está el municipio de Navolato con una cantidad de 39 sectores en los cuales desarrolla sus actividades económicas mismos correspondientes al $12,62 \%$ del total de sectores desarrollados en el estado de Sinaloa, convirtiéndose en el municipio con menor nivel de diversidad según los 5 municipios analizados.

Los resultados anteriores nos indican que el municipio de Culiacán es el que desarrolla más actividades económicas en el estado de Sinaloa, al especializarse en un $42,39 \%$ de los sectores del estado norteño, y el municipio con menor especialización de sectores es Navolato con tan solo $12,62 \%$ lo que corresponde a 39 estados de los 309 totales que se llevan a cabo en Sinaloa.

En cuanto a la segunda variable que determina la complejidad económica, los resultados de ubicuidad los cuales indica que tan imitable es un proceso productivo, es decir, cuántos municipios de los analizados (5) tiene los recursos y capacidades para especializarse en los mismos sectores que desarrolla un municipio, este resultado promedio proviene de la sumatoria de los municipios que también presentan una VCR en un sector en específico, los resultados se presentan en la tabla 2 .

Tabla 2: Ubicuidad de los municipios estudiados.

\begin{tabular}{cc}
\hline Municipio & Ubicuidad \\
\hline Ahome & 1,5357 \\
Culiacán & 1,5124 \\
Mazatlán & 1,5339 \\
Guasave & 1,5529 \\
Navolato & 1,5539 \\
\hline Fuente: Elaboración propia.
\end{tabular}

Para el caso de ubicuidad, la interpretación de estos resultados son diferentes a diversidad ya que la escala para las puntuaciones de esta variable va de 1 a 5 al ser 5 los municipios analizados, sin embargo, la puntuación de 5 es la cantidad mayor de ubicuidad, es decir, los 5 municipios son capaces de especializarse en la misma actividad económica, de caso contrario, la puntuación de 1 es la cantidad de menor ubicuidad, lo cual indica que solo 1 municipio es capaz de especializarse en una actividad económica, la ubicuidad disminuye conforme menos sean los municipios que presentan una VCR en un sector en específico.

El municipio que presenta menor ubicuidad es Culiacán con una cantidad promedio de 1.5124, este es el municipio que mayor especialización presenta en sus procesos productivos, posteriormente está el municipio de Mazatlán con 1,5339 como puntuación de ubicuidad, después está el municipio de Ahome con una puntuación de 1,5357, siguiente está el municipio de Guasave con 1,5529 como ubicuidad y por último está el municipio de Navolato con una puntuación promedio de 1,5539 en ubicuidad.

La complejidad económica es determinada mediante las variables diversidad y ubicuidad, mediante la metodología propuesta por Hausmann e Hidalgo (2009) es posible deducir que mientras mayor sea la diversidad que presenta una entidad económica mayores niveles tendrá de complejidad $\left(\mathrm{K}_{\mathrm{s}, 0}\right)$ y conforme menor ubicuidad tenga en los sectores en los cuales se especializa mayor será su nivel de complejidad $\left(\mathrm{K}_{\mathrm{s}, 1}\right)$. Cuando una economía es 
capaz de aprovechar óptimamente todos sus recursos y de desarrollar sus capacidades, principalmente del capital intangible debido a que de este proviene la transferencia de conocimiento productivo dentro de una economía el cual es necesario para la generación de nuevos productos mediante la innovación en el mercado. Los resultados graficados de cada uno de los municipios se muestran en la gráfica 1 .

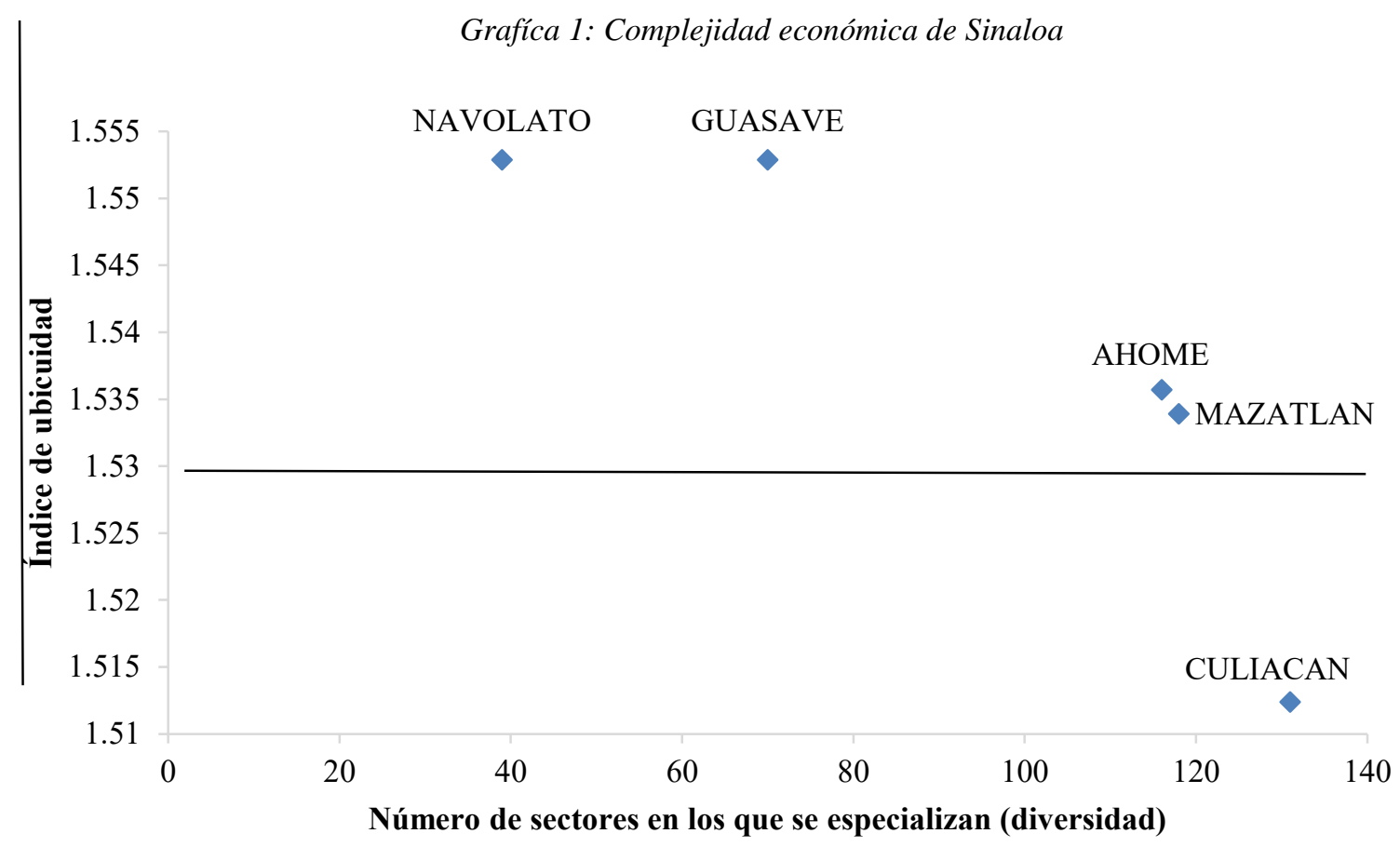

Fuente: Elaboración propia.

En la gráfica 1 se muestra la ubicación de los municipios analizados en relación con su nivel de complejidad económica, donde el eje de $\mathrm{Y}$ está representado por la variable ubicuidad y el eje de las X está representado por la variable diversidad. Conforme más alejado esté la ubicación del municipio del eje de las Y quiere decir que posee mayor diversidad o sectores en los cuales se especializa, mientras más cerca esté del eje de las $\mathrm{X}$ quiere decir que posee menor ubicuidad o que menos cantidad de municipios pueden desempeñar sus actividades económicas en las cual se especializa.

Aquellos municipios que se encuentren en el cuadrante superior izquierdo son los municipios con menor nivel de complejidad, ya que son los que menor cantidad de sectores especializados tienen y la complejidad de sus procesos productivos no es muy alta por lo tanto la mayoría de los municipios pueden realizarlos, que en este caso son Navolato y Guasave. Los municipios que estén ubicados en el cuadrante inferior derecho son aquellos con mayor nivel de complejidad, ya que poseen mayor cantidad de actividades económicas en las cuales se especializan y la complejidad de su estructura productiva es muy alta gracias a la mayor aplicación de conocimiento por lo tanto son pocos los municipios que también pueden realizar dichas actividades, convirtiéndolos en municipios más competitivos, los cuales son Ahome, Mazatlán y Culiacán.

Con base a los resultados del cálculo de las variables se obtuvieron los niveles de complejidad de los municipios analizados los cuales se muestran en la tabla 3.

\section{Tabla 3: Clasificación de complejidad económica de los municipios}


estudiados.

\begin{tabular}{cc}
\hline Municipio & Complejidad Económica \\
\hline Culiacán & 1 \\
Mazatlán & 2 \\
Ahome & 3 \\
Guasave & 4 \\
Navolato & 5 \\
\hline
\end{tabular}

Fuente: Elaboración propia.

Culiacán con valores de 131 en diversidad y 1,5124 en ubicuidad es el municipio con mayor complejidad económica es su estructura productiva obteniendo el primer puesto de los 5 municipios analizados, siguiente en segundo lugar esta Mazatlán con 118 en diversidad y 1,5339 en ubicuidad, próximo en tercer puesto se encuentra Ahome con 116 en la variable diversidad y 1,5357 en la variable ubicuidad, en cuarto puesto está Guasave con 70 en diversidad y 1,5529 en ubicuidad y por último en quinto puesto como el municipio menos competitivo de los analizados esta Navolato con 39 en diversidad y una puntuación en ubicuidad de 1,5539.

\section{CONCLUSIONES}

Partiendo de la teoría y analizando los resultados anteriores, se puede apreciar que con base a Hausmann e Hidalgo (2009) se determina la complejidad económica de los municipios mediante el cálculo las variables diversidad y ubicuidad que conforme a los autores, sirve como una herramienta se auxilia en la toma de decisiones del comportamiento económico de las economías, cumpliendo con la función de clasificador de competitividad y desempeño de las entidades económicas analizadas permitiendo establecer la tendencia de crecimiento de las economías.

Ninguno de los municipios analizados es capaz de especializarse en todos los sectores que se practican en el estado de Sinaloa, el más cercano a lograr esto es el municipio de Culiacán con un valor en diversidad de 131 sectores en los cuales se especializa, que con una puntuación de 1,5124 en ubicuidad, se determina como el municipio con mayor complejidad económica y el mayor competitivo en cuanto a su desempeño de aprovechamiento de recursos mediante la implementación de VCR en su economía. El municipio menos competitivo conforme a los analizados en esta investigación es Navolato ya que solo practica el $12,62 \%$ del total de actividades económicas que se practican en el estado, esto puede ser causado por los mismos supuestos de la teoría de recursos y capacidades de Penrosee (1959) que argumenta que la diferencia entre las entidades económicas es establecida por la nula equidad de posesión de recursos y desarrollo de capacidades productivas.

De los 5 municipios analizados, sólo Culiacán, Mazatlán y Ahome pueden considerarse con un nivel de complejidad alto conforme a sus valores en las variables diversidad y ubicuidad, los municipios Guasave y Navolato son considerados como municipios con un bajo nivel de complejidad

económica con base a los resultados de las mismas variables y su posicionamiento en la gráfica de complejidad económica.

Los municipios que poseen un alto nivel de complejidad económica poseen una oportunidad de crecimiento económico, ya que conforme a los postulados de la teoría de complejidad económica estas economías son las que aplican mayor conocimiento en sus procesos productivos lo cual los determina como complejos, complejos de imitar por parte de la competencia lo cual genera una ventaja competitiva ante el mercado estatal e incluso regional.

Los resultados encontrados en la investigación son de gran impacto para tres diferentes grupos, el gobierno puede observar el comportamiento económico de los municipios más representativos de Sinaloa que al basar los cálculos en el personal ocupado (empleo) puede determinar en qué actividades económicas se concentra la mayor cantidad de capital humano por municipio, de esta forma puede establecer reformas, iniciativas o planes sociales para incentivar aquellos sectores poco desarrollados y de igual 
forma, aprovechar aquellos sectores en los cuales se presentan una VCR. Asimismo, los resultados permiten hacer la recomendación de la generación de nuevas políticas públicas las cuales están enfocadas en explotar las ventajas competitivas de cada uno de los municipios, ya que esto puede ser una herramienta importante para incrementar la competitividad del municipio, las políticas públicas deben estar enfocadas en la aplicación de planes de presupuesto y desarrollo para aquellas empresas que pertenecen a aquellos sectores que presentaron una ventaja competitiva ante los demás municipios en función al personal ocupado. Los inversionistas pueden detectar en qué sectores existe mayor competitividad a nivel estatal conforme a las puntuaciones de ubicuidad, asimismo, conocer qué sectores son áreas de oportunidad para emprender al no existir competencia en el mercado. Y por último la comunidad científica al generar nuevo y productivo conocimiento donde se analiza el comportamiento económico de las entidades económicas más importantes del estado de Sinaloa bajo la perspectiva de la teoría de complejidad económica, creando nuevos indicadores de desempeño bajo estos supuestos que pueden ser hincapié para futuras investigaciones por académicos y universidades. 


\section{Referencias}

Donoso, V., \& Martín, V. (2017). Complejidad económica y densidad productiva como fuentes de competitividad. Estudios de economía aplicada, 35(1), 85. https://dialnet.unirioja.es/servlet/articulo?codigo $=5798830$

García, V M., Meza, L. C., \& Pedraza, F. (2018). Desarrollo de la capacidad dinámica de absorción de conocimiento entre empresas familiares y no familiares de Bucaramanga, Colombia. Revista Lebret. 10, 89-109. https://doi.org/10.15332/rl.v0i10.2199

García, V., Ruiz, M., López-Parra, M. y Placencia, I. (2019) Diversidad y ubicuidad como determinantes de la complejidad económica de los municipios de Sonora. Emprendimiento e innovación empresarial. México: HESS S.A. DE C.V. Tomo 3 (585 - 624) ISBN: 978-6079011-76-5. http://remineo.org/repositorio/memorias/ciao/xviciao/aprendizaje-conocimientoe-innovacion/diversidad-y-ubicuidad-como-determinantes-de-la-complejidad-economica-delos-municipios-de-sonora-mexico/

Gómez, M., Chávez, M. y Mosqueda, M. (2016). Complejidad Económica y Crecimiento Regional, Evidencia de la Economía Mexicana. Documento de investigación. Banco de México. http://www.banxico.org.mx/publicaciones-y-discursos/publicaciones/documentos-deinvestigacion/banxico/\%7B9FED6962-7D52-0C58-DD95-5C33AAE814C3\%7D.pdf

Gómez Zaldívar, Fernando, \& Molina, Edmundo. (2018). Zonas Económicas Especiales y su impacto sobre el desarrollo económico regional. Problemas del desarrollo, 49(193), 1132. https://doi.org/10.22201/iiec.20078951e.2018.193.61285

Hausmann, R. \& Hidalgo, C. (2009). The Building Blocks of Economic Complexity.” the United States of America 106 (26), pp. 10570-10575. Proceedings of theNational Academy of Sciences.

Hausmann, R., Cheston, T., \& Santos, M. A. (2015). La Complejidad económica de Chiapas. BibTex Tagged XML Descargado cid_wp_302.pdf, 3. http://www.tinyurl.com/mzhkh2f

Instituto Mexicano Para Competividad (2016). Un puente entre dos Méxicos: Índice de Competividad Estatal. (INCO) Edición 10(1): 14-20. http://imco.org.mx/wp-content/uploads/2016/11/2016ICE-Boletin.pdf

Instituto Nacional de Estadística, Geografía e Informática (2019) Encuesta nacional de ocupación y empleo

(ENOE). https://www.inegi.org.mx/contenidos/programas/enoe/15ymas/doc/resultados_ciudades_enoe 2019 trim1.pdf

Instituto Nacional de Estadística, Geografía e Informática (2015). Producto Interno Bruto por entidad federativa.

http://www.inegi.org.mx/saladeprensa/boletines/2016/especiales/especiales2016 12 02.pdf

Instituto Nacional de Estadística, Geografía e Informática (2013). Sistema de cuentas nacionales de México. Cuentas a corto y largo plazo. Fuentes y metodología. http://www.inegi.org.mx/est/contenidos/proyectos/cn/pibt/doc/scnm_metodologia_01.pdf

Lara Rodríguez, J., Tosi Furtado, A., \& Altimiras-Martin, A. (2018). Materias primas críticas y complejidad económica en América Latina. Apuntes Del Cenes, 37(65), 1551. https://doi.org/10.19053/01203053.v37.n65.2018.5426

Nonaka, I., y Takeuchi, H. (1995). The knowledge-creating company: How Japanese companies create the dynamics of innovation. Oxford university press.

Programa para Democratizar la Productividad (2015). Atlas de la complejidad económica de México. Gobierno Federal. https://www.gob.mx/productividad/articulos/atlas-de-complejidadeconomica-de-mexico-14291?idiom $=$ es

Pérez Hernández, C. C., Moheno Mendoza, J., \& Salazar Hernández, B. C. (2019). Análisis estadístico de la capacidad de absorción en México y su influencia en la generación de conocimiento tecnológico. Innovar, 29(72), 41-58. http://www.scielo.org.co/pdf/inno/v29n72/0121-5051-inno-29-72-41.pdf

Pérez Hernández, Carla Carolina, Salazar Hernández, Blanca Cecilia, \& Mendoza Moheno, Jessica. 
(2019). Diagnóstico de la complejidad económica del estado de Hidalgo: de las capacidades a las oportunidades. Revista mexicana de economía y finanzas, 14(2), 261-277. https://doi.org/10.21919/remef.v14i2.299

Sánchez Peñaflor, S; Herrera Avilés, M. (2016). Los recursos humanos bajo el enfoque de la teoría de los recursos y capacidades. Revista Facultad de Ciencias Económicas: Investigación y Reflexión, XXIV, 133-146. Colombia. http://www.redalyc.org/articulo.oa?id=90947653008

Tortoriello, M. (2015). The social underpinnings of absorptive capacity: the moderating effects of structural holes on innovation generation based on external knowledge. Strategic Management Journal, 36(4), 586-597. http://dx.doi.org/10.1002/smj.2228

Vega-Jurado, J. y Schmutzler, J. (2017). Determinantes del uso de fuentes externas de conocimiento en los procesos de innovación empresarial: la importancia de la dimensión regional. Espacios, 38(19), 23-41.

Vargas Hernández, J. G \& Cano Ibarra, S. T. (2016). Evaluating higher education institutions through agency and resources -capabilities theories. A model for measuring the perceived quality of service. Independent Journal of Management \& Production, 7() 1126-1153. http://www.redalyc.org/articulo.oa?id=449549377008 